\title{
„Geschenke für alle“. Über Die 3 Tornados und die Berliner Gegenkultur
}

Hans-Jochen Krank-Hover, Ex-Tornado, im Gespräch mit Andreas

Häcker.

Biesenthal, Brandenburg, 19. Juli 2012

Andreas Häcker et Hans-Jochen Krank-Hover

\section{(2) OpenEdition}

\section{Journals}

Édition électronique

URL : https://journals.openedition.org/ceg/8991

DOI : 10.4000/ceg.8991

ISSN : 2605-8359

Éditeur

Presses Universitaires de Provence

Édition imprimée

Date de publication : 1 mai 2013

Pagination : 297-306

ISBN : 0751-4239

ISSN : 0751-4239

Référence électronique

Andreas Häcker und Hans-Jochen Krank-Hover, , Geschenke für alle“. Über Die 3 Tornados und die Berliner Gegenkultur", Cahiers d'Études Germaniques [Online], 64 | 2013, Online erschienen am: 02 Januar 2020, abgerufen am 02 Juni 2021. URL: http://journals.openedition.org/ceg/8991 ; DOI: https://doi.org/10.4000/ceg.8991 


\title{
„Geschenke für alle“" Über Die 3 Tornados und die Berliner Gegenkultur
}

\author{
Hans-Jochen Krank-Hover, Ex-Tornado, \\ im Gespräch mit Andreas Häcker \\ Biesenthal, Brandenburg, 19. Juli 2012
}

Andreas Häcker: Was wolltet ihr als 3 Tornados machen: Kabarett, Kleinkunst, Liederabende?

Hans-Jochen Krank-Hover: Wir wollten also kein Kabarett machen in der Form, wie es bis Mitte der siebziger Jahre die etablierten Kabarettisten gemacht haben. Deswegen haben wir uns auch nicht „Kabarett" genannt. Wenn du dir die erste Platte anschaust, siehst du auf dem Cover „Straßentheater, Tingeltangel, Musiktheater, Kneipentheater, Kneipenkabarett ${ }^{c .}$... wir haben versucht dafür einen neuen Begriff zu finden. Also wir wollten nicht so arbeiten wie das literarische Kabarett. Da haben sich die Leute auf die Bühne gestellt, haben vielleicht einen Dialog geführt und dann ein paar Headlines aus der BILD-Zeitung umgeformt und darüber Witze gemacht. Die haben auch vielleicht ein bisschen gesungen, aber das war doch auch immer sehr statisch und starr. Das war der eine Aspekt, also dass man sich formal abgrenzt von denen. Der zweite Aspekt war, dass wir uns auch inhaltlich abgrenzten. Das kam von Arnulf und Günter, die haben schon vorher, d. h. vor der Gründung der Tornados, Auftritte gehabt, Stücke geschrieben und entwickelt. Das fand ich einfach toll während der Unistreiks 1977. Wichtig war also, dass wir uns auch inhaltlich abgrenzten und nicht diese ewigen Witze über die etablierten Politiker machten wie die Hofnarren. Eigentlich ist das politische Kabarett immer der Hofnarr gewesen für die Politiker. Ich weiß noch, da saß bei der Münchner Lach- und Schießgesellschaft Willy Brandt immer in der ersten Reihe und hat auch über die Witze gelacht, die sie über ihn machten. Wir wollten ein Teil der linken Bewegung sein, des linken Spektrums, die Bewegung unterstützen, zum Beispiel die Bürgerinitiativen, dann gab es die Hausbesetzerbewegung, die Schwulenbewegung, die Frauenbewegung, das war ja endlos, dann diese 
Landkommunenbewegung, die Bewegungen insgesamt, die neue Lebensformen ausprobiert haben. Unser Ziel war es die alle zu unterstützen, indem wir Stücke gemacht haben, zum Beispiel gegen den Autobahnbau. Wir haben ein Stück gemacht für die Gegner des Autobahnausbaus. Dabei haben wir immer in den Stücken versucht, solche Richtungen, wenn sie dogmatisch wurden, wenn ein gewisser Dogmatismus entstanden ist in der Frauenbewegung oder auch in den anderen Bewegungen, also wenn das übertrieben war, wenn extreme Positionen bezogen wurden, das $\mathrm{zu}$ durchbrechen und haben das immer gebrochen. Durchbrochen durch den Witz, durch Ironie, durch Zynismus. Wir wollten auch über uns selbst lachen, über unsere eigenen moralischen Ansprüche, die wir damals stellten und teilweise verabsolutiert haben. Die ersten Hauptgegner, unsere Hauptkritikpunkte, waren diese K-Gruppen, diese kommunistischen, marxistischen, leninistischen Gruppen, die sich ständig neu gebildet haben: ML-Gruppen, Kommunistischer Bund Westdeutschlands, KPDs. Die waren ja schon in der Auflösung begriffen. Mit dem Stück Die Osterinsel haben sie dann den letzten Kick bekommen, so dass sie von der Weltbühne abdanken mussten. Ein anderer wichtiger Punkt war der Terrorismus. Die Bundesregierung und zahlreiche Politiker haben doch viele Linke verdächtigt, dass sie Sympathisanten der RAF sind. Wir haben dann durch unser Auftreten doch versucht, Ängste zu nehmen und dem auch etwas entgegenzusetzen.

AH: Kommen wir zu den Künstlern der 3 Tornados. Ihr seid aus allen Ecken Westdeutschlands nach Berlin gekommen.

HJKH: Arnulf kam aus dem Ruhrgebiet, aus Wuppertal, Günter kam aus Celle. Ich kam aus Frankfurt am Main. Ich bin 1971 nach Berlin gekommen, weil ich nicht zur Bundeswehr wollte. Ich weiß jetzt gar nicht mehr, wie es bei den beiden anderen jetzt war. Das könnte auch so sein, dass sie auch deswegen nach Berlin gekommen sind. Berlin war für so einen Westdeutschen doch eine neue Erfahrung. Das Leben war hier in Berlin viel mehr politisiert, es gab viel mehr Bewegung, es ist auch viel kreativer gewesen hier in Westberlin, in der Großstadt. Ich war fasziniert von Westberlin und habe gedacht, hier könnte man doch was machen. Ich habe zuerst einmal studiert, und dann ging es immer von einem Streik zum nächsten. Es war immer sehr politisch. Die Universität, die einzelnen Veranstaltungen, die Vorlesungen, die Seminare wurden ja teilweise von uns in den Semesterferien vorbereitet, damit man dem Professor sagen kann, was wir denn überhaupt lernen wollen. Das war doch eine ganz andere Welt in Berlin. Das war einfach toll.

AH: 1971 kommst du nach Berlin, lebst in Wohngemeinschaften.

HJKH: Damals gab es nicht so viele Differenzierungen in den verschiedenen politischen linken Richtungen. Es gab zwar schon die 
Trotzkisten, die KPD-MLer, die Anarchisten, aber die waren alle noch zusammen. Am Anfang, als ich nach Berlin kam, habe ich mit Leuten zusammengewohnt, die dann später in die RAF gingen und dann verschwunden sind, die untergetaucht sind. Das hat sich danach vollkommen zersplittert, verhärtet in den einzelnen Teilen. Und erst die Alternativbewegung hat es geschafft, etwas Neues entstehen zu lassen. Und wir Tornados sind dann sozusagen mit geschwommen. Beziehungsweise haben wir sie mit angetrieben.

AH: Du kommst aus Frankfurt und hast dort das Ende der 60er Jahre erlebt.

HJKH: Ich war Lehrling, Laborant bei den Farbwerken Hoechst und habe am Hessenkolleg in Rüsselsheim mein Abitur nachgemacht. Da war ich von 1968 bis 1971. Und da bin ich auch schon sehr politisiert worden. Da haben wir auch schon ein halbes Jahr lang gestreikt am Hessenkolleg, um mehr Rechte durchzusetzen für die Kollegiaten und die Schüler, um mehr Einfluss nehmen zu können auf die Inhalte, auf die Lehrpläne, bis hin zur Teilnahme an Konferenzen, Einflussnahme auf die Notengebung. Da war auch schon viel los zu dieser Zeit.

AH: Wie bist du von der Fabrik an die Universität gekommen?

HJKH: Als ich gearbeitet habe, standen die Studenten des Sozialistischen Studentenbundes (SDS) an den Fabriktoren und haben Flugblätter verteilt, um die Leute zu informieren über den Vietnamkrieg, die „Trikontinentaltheorie“" . Es gab Che Guevara, Aufbruch in der Dritten Welt, Revolution... ich muss gestehen, ich habe damals nichts verstanden. Ich habe die Flugblätter nicht verstanden, ich bin auf Veranstaltungen vom SDS gegangen, ich habe mir gesagt, da musste du mal hingehen, das wäre interessant, was die da so erzählen. Ich muss sagen, ich habe nichts verstanden. Da wusste ich, da müsste ich noch mal zur Schule gehen und mein Abitur machen, ich blicke da nicht durch. Das war eigentlich so die Haupttriebfeder weiterzumachen, Abitur zu machen und dann zu studieren.

AH: Wie war das im Jahr 1977, als ihr mit den 3 Tornados anfingt? Wie kommst du zum Trio?

11966 fand eine „Trikontinentale Konferenz“ in Havanna statt, die sich der Frage der Dekolonisierung und des bewaffneten Widerstands gegen den Imperialismus widmete. In einer „Botschaft an dic Trikontinentale Konferenz" schricb Che Guevara: „Schaffen wir ein, zwci, viele Victnam". Es wurde cine von der internationalen Anti-Victnamkricgsbewegung (auch von der deutschen Protestbewegung) immer wieder aufgegriffene Parole (Alle Anmerkungen von Andrcas Häcker). 
HJKH: Ich habe Arnulf und Günter bei den Unistreiks 1977 gesehen, hatte die beiden in der Kneipe kennengelernt. Über Freunde, die auch in der Szene waren, bin ich mit den beiden zusammengekommen. Sie haben bei den Unistreiks Sketche gespielt. Da dachte ich, da könnte ich doch mitmachen. Die beiden haben die Idee auch aufgegriffen. Da gab es dann auch noch ein Treffen beim Schauspieler Otto Sander, und zwar hatte Günter an der Schaubühne als Regieassistent gearbeitet und hatte Kontakte zum Theater ${ }^{2}$. Und dann habe ich mein Akkordeon mitgenommen, ich habe ja schon immer Akkordeon gespielt, so Blödellieder, alte Schlager, auch in der Kneipe habe ich immer Musik gemacht. Ich weiß es nicht mehr so genau, aber ich musste da bei Otto Sander eine Einlage spielen und Otto Sander hat gesagt: „Das ist der richtige Mann, der da bei euch mitmachen kann.“ (Lacht.) Die beiden haben dann gesagt, okay, wir machen das. Und so haben wir dann angefangen. Aber da waren wir noch nicht die 3 Tornados, wir mussten uns den Namen erst noch überlegen.

AH: Wie kommt es zum Namen Die 3 Tornados? Warum nicht Kampfkabarett?

HJKH: Wir wollten gerade nicht so martialisch politisch sein im alten Stil. Wir wollten nicht die Stadtguerilla, sondern die "Spaßguerilla“ als Alternative. Arnulf und Günter hatten ja schon tolle Ideen gehabt, und haben zu jedem Flugblatt einen Mohrenkopf verteilt, damit es auch gelesen wird. Es sollte schon etwas Witziges sein. Wir saßen zusammen und fanden nicht so richtig einen Namen. Da waren die beiden, das sind meine Erinnerungen, auf einem Volksfest, das war wohl das deutsch-französische Volksfest, glaube ich, und da hat Günter irgendwo das gesehen: die Tornados. So kam es zu den 3 Tornados.

AH: Welche Beziehungen hattet Ihr zur institutionalisierten Kultur? Günter Thews arbeitete beim Fernsehen, jeder von euch hatte einen Job oder studierte.

HJKH: Ja, Günter war Regieassistent in Hamburg beim Fernsehen, arbeitete bei Aufnahmen für Tatort und so. Er wollte ja auch beim Fernsehen einsteigen. Aber als wir dann mit den Tornados immer mehr Erfolg hatten, musste er sich entscheiden. Er hat sich schließlich für die Tornados entschieden und da haben wir so richtig losgelegt. Der Günter war ja der Hauptmotor, er hat viele Stücke geschrieben und viel Energie reingesteckt. Entstanden sind die 3 Tornados aus den Unistreiks 1977, dann die Unterstützung der Streiks. Günter und Arnulf waren Theaterwissenschaftler. Dario Fo, der damals oft nach Berlin kam, war ein großes Vorbild, ebenso das Stcin.

2 Otto Sander gehörtc seit 1970 als Schauspicler zum Schaubühne-Enscmblc um Peter 
The San Francisco Mime Troupe ${ }^{3}$. So kamen sie auf die Idee, selbst auch Theater zu machen, Sketche zu schreiben, um die linken Bewegungen zu unterstützen. Wir mussten uns entscheiden: machen wir das nun voll oder nicht? So wurde das dann professionell. Ich war Student und Taxifahrer, um mein Studium zu finanzieren. Ich habe noch während der Tornado-Zeit mein Graecum nachgemacht, weil ich noch in Philosophie promovieren wollte und hatte schon mein Exposé eingereicht. Ich bin von einem Auftritt aus Wien zurückgeflogen, um dann 1978 oder 1979 in Steglitz mein Graecum nachzumachen. Ich müsste mal nachgucken. Das ist dann das letzte, was ich an der Uni gemacht hatte.

AH: Die 3 Tornados waren ständig auf Tournee. Nicht nur in Berlin, sondern quer durch die ganze Republik.

HJKH: Ja, die Hälfte des Jahres waren wir unterwegs. Wir hatten 120 bis 150 Auftritte pro Jahr. Wir kamen bis Südtirol, in Österreich waren wir viel, in der Schweiz und einmal sogar in Holland. Immer wurden wir von Leuten eingeladen. In der Schweiz lief das zum Beispiel über Pinkus, eine Art Landbewegung. Wir wurden überall eingeladen, wo es alternative Bewegungen gab. Wir haben übrigens ganz viel Sketche gemacht für den Österreichischen Rundfunk, für eine Jugendsendung, die haben ganz viele kleine Sketche aufgenommen. Die Sketche würde ich mir gern noch einmal anschauen. Wir sind oft nach Wien gefahren. Das war sehr minimalistisch, wir wurden da vor einer weißen Wand aufgenommen.

AH: In manchen Bühnennummern wie dem Saint-Tropez-Twist oder dem Schwarzseher-Lied (GEZ-Song) wird viel getanzt. Hat Euch Fred Astaire inspiriert? Goldene gestreifte Hosen als Hommage an den Meister?

HJKH: Das ist ja Quatsch. Die goldenen gestreiften Hosen haben wir nur einmal getragen, wir haben sie extra für einen Auftritt anfertigen lassen. Leider sind die Hosen heute verschwunden. Natürlich haben wir für die Musik populäre eingängige Melodien genommen, dazu aber neue Texte. Sehr beeinflusst hat mich Joe Hill, der Gewerkschaftler aus den USA ${ }^{4}$. Ich hatte so ein Joe-Hill-Liederbuch aus Schweden. Wir haben dann aber auch Showelemente verwendet, um den Vortrag der Lieder aufzupeppen.

3 Politische Straßentheatergruppe, die 1959 in San Francisco gegründet wurde und sich mit den Mittcln der Commedia dell'arte, des Politschwanks oder des Melodramas für dic Bürgerrechtsbewegung einsetzte oder gegen dic Aktivitäten des amerikanischen Militärs protesticrtc.

4 Joc Hill (1879-1915) war cin aus Schweden stammender, amerikanischer Wanderarbeiter, Gewerksehaftsaktivist und Folksongs-Autor. Des zwcifachen Mordes angeklagt, wurde er trotz mangelnder Beweise 1915 hingerichtet. Scin Fall wurde zu einem der größten Skandale der amcrikanischen Justizgeschichte. 
AH: Anders formuliert: ist die Steppeinlage à la Fred Astaire eine subversive Parodie kommerzieller Hochkulturmodelle?

HJKH: (Lacht.) Wir haben uns ja mehr als Subkultur verstanden, als die andere Kultur. Die andere Kultur im Unterschied zur etablierten Kultur. $\mathrm{Ob}$ das jetzt hoch oder tief ist, weiß ich jetzt gar nicht $\mathrm{zu}$ beurteilen. Aber natürlich musst du, wenn du die Leute erreichen willst, solche Sachen benutzen. Man braucht eingängige Muster. Wir haben dann auch so Strukturen von Fernsehsendungen übernommen und angefangen, sie umzuformen. Wir haben das verwendet, was jeder kennt. Darauf musst du aufbauen, um die Leute zu erreichen.

AH: In euren Show-Programmen findet man bewusst gefälschte, parodierte Radiosendungen oder Tagesschauen. Inwiefern war das antiautoritär?

HJKH: Immer. (Lacht.) Wir waren schließlich ein Teil der antiautoritären Bewegung. Es musste also auch immer so sein, dass man mit Hilfe von Witz, Parodie und Ironie autoritäre Auswüchse, die auch in der linken Bewegung da waren, bricht, abkappt und immer aufzeigt mit Hilfe von Witz, so wie die Spaßguerilla: „Hier geht ihr zu weit!“”. Das war antiautoritär auch innerhalb unserer eigenen Bewegung.

AH: Eine Frage zu Eurem Geschäftsmodell. Einerseits spieltet Ihr gratis auf linken Benefizveranstaltungen, andererseits in großen subventionierten Stadttheatern.

HJKH: Zum Beispiel Claus Peymann, der in Bochum Theaterdirektor war, fand uns gut und hat uns eingeladen. Da gab es dann richtige Gagen. Wir traten auch im Mainzer Unterhaus auf. Wenn man in einem richtigen Theater auftrat, bekam man richtige Einkünfte, man wurde prozentual an den Einnahmen beteiligt. Oder auch in der Markthalle in Hamburg. Da kam dann richtig gut Geld rüber und wir konnten dann auch mal umsonst spielen oder einen Hut rumgehen lassen. Es war aber immer genug Geld da.

AH: Ihr seid eher eine Live-Gruppe gewesen. Welche Rolle spielten für euch die Langspielplatten?

HJKH: Der Vorschlag, eine Langspielplatte zu machen, kam vom Münchner Trikont-Verlag5, das fanden wir schon sehr gut. Nur lief der

5 Legendärer Gegenkultur-Verlag, der 1967 von zwci SDS-Studenten gegründet wurde und eng mit der sogenannten ,Sponti-Szene“ verwoben war. Er trat u. a. mit Publikationen über dic Befreiungskämpfe in der Dritten Wclt, übcr dic amcrikanische „Black Panthers“-Bewegung und über den Feminismus auf. Er veröffentlichte auch Schriften, die sich von den Kampfmethoden der RAF nachdrücklich distanzicrten. 
Plattenverkauf nicht so richtig, die Auflagen waren nicht so hoch, das lag vielleicht auch daran, dass es nicht so berauschend war, das Programm nur zu hören. Auch die Qualität war nicht so gut, vielleicht mag es auch daran gelegen haben. Die Auflage war wirklich gering. Wir haben teilweise Platten auch verschenkt. (Lacht.)

AH: Im Online-Archiv der Tornados kann man heute auch eine Kassettenaufnahme von Euch von 1980 herunterladen. Die Kassette war damals das Medium, das man heute mit der mp3-Datei vergleichen kann.

HJKH: Damals gab es in der Tat Verlage, die voll und ganz auf die Kassetten gesetzt haben, weil in jedem Zimmer ein Kassettenrekorder stand. Es gibt eine Aufnahme von einem ganzen Tornado-Konzert in Freiburg. Die Kassette ist eine echte Rarität. Ich muss das Cover mal auf die Webseite stellen.

AH: Früher gab es keine Smartphones, E-Mail und soziale Netzwerke im Internet. Die 3 Tornados waren Netzwerker innerhalb des linken alternativen Milieus, die „lebendige Zeitung“, die sagt, was im Land los ist.

HJKH: Es gab auch noch andere Informationsmittel, linke Zeitschriften. Uns als „Netzwerker“ zu bezeichnen, ist vielleicht ein bisschen übertrieben. Natürlich haben wir z. B. für die tageszeitung in ganz Deutschland und in der Schweiz eine Tournee gemacht, um für die Gründung der taz zu werben. Von daher haben wir das schon verbreitet und publik gemacht.

AH: Kommen wir zum Thema Kneipe und Musik. Was waren eure Bühnenerfahrungen vor den 3 Tornados?

HJKH: Ich hatte schon Bühnenerfahrungen als Rockmusiker mit den Shatters. Ich habe jahrelang gespielt. Auf der Bühne, in Bars. Jedes Wochenende: Musikmachen. Gitarre, Saxophon habe ich gespielt. Als Musiker auf der Bühne musst du immer gucken, was das Publikum will. Das war meine einzige Erfahrung. Aber die habe ich abgebrochen, als ich nach Berlin bin. Ich habe meine ganze Anlage, meine Musikinstrumente verkauft und wollte dann studieren und was Neues machen. Inwiefern Arnulf und Günter Vorerfahrungen hatten, weiß ich jetzt nicht. Außer du nimmst jetzt die Kneipe als Bühne, da musst du immer eine kleine Aufführung machen.

AH: Die Berliner Eckkneipe ist ein mythischer Ort. Inwiefern war die Kneipenszene, das „Godoth“ oder der „Schlemihl“, ein Treffpunkt der undogmatischen Linken?

HJKH: Die Kneipe war das verlängerte Wohnzimmer. Die Eckkneipe, in der wir uns immer getroffen haben, war die "Mautze“, Ecke 
Grünewaldstraße/Elßholzstraße in Schöneberg, die gibt es heute nicht mehr. $\mathrm{Da}$ trafen sich also alle aus meinem Bekanntenkreis. Wenn du jemanden treffen wolltest, sagtest du, treffen wir uns in der Mautze um zehn Uhr, elf Uhr, zwölf Uhr. Da saßen wir dann immer bis nachts um drei. Dann gab es diese Szenekneipen, die sind ja erst ein bißchen später entstanden, wie der „Schlemihl“. Günter war immer viel im „Slumberland“, das wurde dann der „Dschungel“" am Winterfeldtplatz. Dann gab es ein paar andere Kneipen wie der „Leuchtturm“ in der Crellestraße. Ich war in Schöneberg, Günter und Arnulf wohnten in Kreuzberg, Arnulf nahe an der Yorckbrücke, also nahe an Schöneberg. Also ich war ein ständiger Kneipengänger, da traf man dann jeden, den man treffen wollte. Und dann haben wir ja in den Kneipen auch Aufführungen gemacht, auch in der Mautze, obwohl das eine ganz kleine Kneipe war. Oder ein kleiner Auftritt im Dschungel oder im „Anderen Ufer“ in der Hauptstraße, usw.

AH: Wo habt Ihr geprobt? In der Ufa-Fabrik? Musstet Ihr Euch einen Proberaum besetzen?

HJKH: Wir haben immer zu Hause geprobt. Mal bei dem, bei dem, bei dem. Als dann das Ufa-Gelände in Tempelhof besetzt wurde, standen dort so viele Häuser und Räume zur Verfügung. Da habe ich den Juppy gefragt ${ }^{6}$, ob er nicht einen Raum für uns hätte. „Na klar“", hat er gesagt. Und so hatten wir einen Raum zum Proben und zum Lagern der Requisiten. Ansonsten hätten wir uns eine Ladenwohnung mieten müssen, um das Ganze zu schultern.

AH: Wir sprachen vorhin von Spaßguerilla. Einer der Praktiker und Theoretiker der Spaßguerilla war Fritz Teufel. Habt ihr euch da inspiriert?

HJKH: Fritz Teufel, der später auch untergetaucht ist, im Gefängnis gesessen hat und doch unschuldig war, und so den Staat an der Nase herumgeführt hat durch seine eigene List, war einer, der uns sehr inspiriert hat. Oder auch Günter Wallraff, der verschiedene Sachen aufgedeckt hat ${ }^{7}$. Natürlich war der nicht so witzig. Fritz Teufel hatte immer diese humoristische Ader gehabt. Mit Langhans hatte er zum Beispiel ein Buch herausgegeben: „Klau mich!“ hieß es, es wurden ja immer viel Bücher geklaut, das war ja erlaubt (lacht), es gab viele Raubdrucke. „Klau mich“ war doch sehr motivierend.

6 ,Juppy“ ist der Spitzname Hans-Josef Bechers, des berühmtesten Berliner „MultikulturImpresarios" auf dem ehemaligen Gelände des UFA-Kopierwerks, das 1979 von der Kommune Fabrik für Kultur, Sport und Handwerk friedlich besetzt wurde und seitdem selbstverwaltet ist.

7 Wallraff erregte in den siebziger Jahren mit seinen als verdeckter Ermittler geführten Reportagen Aufsehen: 1969 erschien 13 unerwünschte Reportagen, $1973 \mathrm{Ihr}$ da oben - wir da unten und 1977 Der Aufmacher. Der Mann, der bei „Bild“ Hans Esser war. 
AH: Die 3 Tornados waren Musiker, Tourneekünstler. Gab es Sex, Drugs und Rock'n'roll?

HJKH: Sex, Drugs und Rock'n'Roll? Da gab es viel Alkohol, Zigaretten. Sex?

AH: Gab es Groupies?

HJKH: Sex ist ja mehr eine private Sache. Günter war schwul. Er hatte Syphilis gehabt und ist an AIDS gestorben. Er war bei der Schwulenbewegung. Vielleicht ist man da auch ein bisschen unvorsichtig umgegangen beim Sex. Es gab keine Exzesse bei den 3 Tornados.

AH: Nicht wie bei den Rolling Stones?

HJKH: Nein, keine Exzesse. (Lacht.) Nein, auf keinen Fall. Da waren wir sauber.

AH: Die 3 Tornados haben nicht nur Straßen- und Kneipenspektakel produziert. Ihr seid auch unter die Macher des neuen deutschen Films gegangen. Wie heißt euer Werk?

HJKH: Es war ein Kurzfilm: „Geschenke für alle“. Wir spielten die Hauptrollen. Ein Off-Sprecher, eine bekannte Stimme, ein Sprecher, der im Fernsehen die Nachrichten gesprochen hat, erzählt am Anfang, dass es schneit, sich Weihnachten über die Stadt legt, der Kurfürstendamm im Kaufrausch ist und auch in der Tauentzienstraße die Kaufhäuser wieder geöffnet sind, usw. Die Kamera fährt auf eine kleine Gruppe von Mitgliedern der Heilsarmee zu, die dort Musik macht, und dann treten wir auch schon in Erscheinung. Dann kommt es so, dass die Ost-Berliner auch mehr Geschenke haben wollen und sie stürmen die Mauer, durchbrechen die Mauer, stürmen nach Westberlin und kaufen alles weg. Wir sind dann im KaDeWe [Kaufhaus des Westens], ich spiele einen Reporter, interviewe die Leute. Arnulf spielt einen Weihnachtsmann. Günter spielt einen Kaufhausdetektiv oder so etwas. Wir sind vollkommen machtlos gegenüber den Ossis, die alles wegkaufen. Die kaufen im KaDeWe sogar Kohlen. Hauptsache: es ist aus dem Westen! Mit dem Film „Geschenke für alle" haben wir ja die Bewegung schon vorweggenommen, weil 1989, als die Mauer geöffnet wurde, war es ja so, da haben die Leute aus dem Osten im Westen alles weggekauft.

AH: Aus welchem Jahr war der Film? 
HJKH: Das weiß ich jetzt nicht mehr so genau. 1979,1980 oder $1981^{8}$. Auf jeden Fall fängt der Film so an: Weihnachten, in Berlin... 1979, glaube ich, naja, der Film wurde ja jedes Jahr in den alternativen Off-Kinos vor Weihnachten immer als Vorfilm gezeigt. Man hatte die Idee, das Datum immer zu ändern, weil es ja nicht mehr passte. Wir haben den Film in etwa einer Woche gedreht. Arnulf hatte von der Filmhochschule eine richtig große $16 \mathrm{~mm}$-Kamera besorgt. Es ist ein richtiger Kinofilm, kein Video. Das war damals sehr teuer, das Filmmaterial musste bezahlt werden. Die Filmcrew hat aber alles umsonst gemacht ${ }^{9}$.

AH: Mit dem öffentlichen-rechtlichen Fernsehen hat es selten geklappt, es gibt nur wenige Mitschnitte.

HJKH: Zuvor hatten wir schon Kontakte mit dem Süddeutschen Rundfunk in Stuttgart, hatten auch schon angefangen, einen Film fürs Fernsehen zu drehen, einen antiautoritären Film zum 30jährigen Jubiläum des Grundgesetzes. Wir haben da ein paar Szenen aufgenommen, das Projekt wurde aber abgebrochen, weil es denen zu radikal war. Im Fernsehen haben wir auch nichts mehr bekommen. Wir sind aber mit anderen Gruppen zusammen bei größeren Abenden aufgetreten, zum Beispiel mit dem Frankfurter Fronttheater ${ }^{10}$ in der Kölner Oper. Vielleicht gibt es noch ein paar Aufnahmen von den verschiedenen Theaterfestivals.

AH: Jochen, danke für dieses Gespräch.

8 Es war 1980

9 Die ,Filmhochschule“ war die Deutsche Film- und Fernsehakademie, deren Studenten oft politisch-agitatorische Aktionen filmisch unterstützten. Siehe die Beiträge von J. Hamers und A. Combes in diesem Band.

10 Es wurde im Herbst 1976 als kollektives Kabaretttheater der Frankfurter alternativen Szene gegründet und betrieb kabarettistisch-politische Agitation zu aktuellen Anlässen: Hausbesetzungen, Antiatomdemonstrationen usw. 